\title{
ACTITUDES RELACIONADAS CON LA CIENCIA: UNA REVISIÓN CONCEPTUAL*
}

\author{
VÁZQUEZ ALONSO, A. ${ }^{1}$ y MANASSERO MAS, M.A. ${ }^{2}$ \\ t Ministerio de Educación, Palma de Mallorca. \\ 2 Departamento de Psicología, Universidad Islas Baleares.
}

\begin{abstract}
SUMMARY
This study starts from some previous reports about science related attitudes and the main acbievements and contributions to this area along the last decades. The core of these reflections is reviewed here: to define more accurately the attitude objects in science, to obtain more valid and reliable instruments of assessment, the factors and variables correlated with attitudes, the contributions of the Science-Technology-Society movement, and, as a theoretical framework, the attention to the models of attitudes suggested by the Social Psychology. As a synthesis aimed to help science researchers and teachers, a taxonomy of science related attitudes is suggested here to enhance the education of attitudes as a tool to achieve a humanistic scientific literacy in students.
\end{abstract}

\section{ACTITUDES RELACIQNADAS CON LA CIENCIA: UNA REVISION CONCEPTUAL}

A lo largo de más de 30 años se ha dedicado gran cantidad de tiempo y esfuerzos a la investigación sobre actitudes relacionadas con la ciencia, en su más variados aspectos: el efecto de la educación sobre las actitudes, las relaciones causa/efecto entre las actitudes y diversas variables, la validez de las escalas y cuestionarios de evaluación de actitudes y el contenido misno de las actitudes de los alumnos bacia temas relacionados con la ciencia, como la enseñanza de las ciencias, las clases de ciencias, Ia naturaleza de la ciencia, la incidencia de la ciencia sobre la sociedad, las características de los científicos, los temas sociales y ambientales de la ciencia, etc. Sin embargo, sólo se pueden extraer unas pocas y vacilantes conciusiones, debido en la mayoría de los casos a la gran cantidad de resultados contradictorios obtenidos y a los importantes defectos metodológicos de algunas investigaciones (Schibeci, 1984). Casi una década después, otros dos prestigiosos investigadores en el área (Shrigley y Koballa, 1992) vueiven sobre el mismo tema, senalando que la situación sigue pareciđa: falta de un marco teórico que sirva de referencia a las iniciativas investigadoras y defectuosas evaluaciones de las actitudes. Como remedio a esta situación proponen una decisión revolucionaria, en el sentido kuhniano: la psicolo- gía social, disciplina pionera en la investigación teórica y empírica sobre las actitudes, en general, debería ser el marco teórico y la referencia obligada que se busca.

Esta compleja problemática no es sólo propia del área especiffica de las actitudes relacionadas con las ciencias, sino de todo el campo de las actitudes generales que desde su inicio han sido estudiadas por la psicología social, desde las actitudes políticas y sociales a aquellas otras más específicamente educativas. Esta complejicad puede ser puesta de manifiesto por los siguientes datos: en el periodo 1970-79 se contabilizan 20.209 libros y artículos bajo el epígrafe de actitudes en el Psychological Abstracts; por otro lado, las definiciones del término actitud se cuentan por cientos: Ajzen y Fishbein (1977), en su revisión, encuentran 500 definiciones de actitud en 200 estudios, en el 70\% de Ios cuales la actitud se define de más de una manera. Simplificando mucho, la historia de las actitudes a lo largo de las últimas tres décadas, se podría resumir diciendo que: la década de los sesenta fue la del revisionismo y las críticas radicales a los excesos y errores cometidos en la investigación đe las actitudes; durante los setenta se pusieron en práctica análisis y diseños más depurados y estrictos; y en los ochenta se 
han desarrollado los modelos cognitivos de las actitudes como el de la acción razonada (Fishbein y Ajzen, 1981) y el del procesamiento de la información (modelo probabilístico de la información de Petty y Cacioppo, 1986).

Para la mayoría de los profesores, el concepto de actitud se identifica implícita y comúnmente con la disposición de los estudiantes hacia el aprendizaje de la ciencia, y que se operacionaliza en el interés del alumnado por la ciencia, la motivación hacia su estudio, el agrado y la buena disponibilidad que demuestra, la puntualidad en el cumplimiento de las tareas escolares, la atención en clase, etc. En esta concepción reducida subyace un carácter excesivamente instrumental y secundario, es decir, la actitud así concebida es un medio para alcanzar buenos resultados de aprendizaje de Ia ciencia; el inconveniente de esta concepción es que niega la independencia de la actitud como un contenido autónomo de aprendizaje equiparado en un plano de igualdad con los más estimados contenidos conceptuales y de procesos. En esta revisión se pretende sugerir un concepto multidimensional de las actitudes relacionadas con Ia ciencia (en plural), cuya definición precisa y delimitada respecto a otras no sólo sea un paso metodológico necesario para una válida y correcta evaluación de las actitudes, sino también para la aceptación de las actitudes como contenidos independientes e importantes de la educación en ciencias.

\section{ALGUNAS REVISIONES SOBRE ACTITU- DES RELACIONADAS CON LA CIENCIA}

En el reducido espacio de este artículo se hace difícil resumir los rasgos pormenorizados de la ingente, prolífica y compleja investigación sobre actitudes relacionadas con la ciencia, de modo que la revisión que aquí se ofrece tomará como punto de partida los resultados principales de algunas revisiones cruciales previas y bient conocidas, para describir después, con más detalle, algunos trabajos recientes, posteriores a esas revisiones, y especialmente relevantes para el tema de las actitudes relacionadas con la ciencia.

La primera gran revisión realizada por Ormerod y Duckworth (1975) sobre 500 trabajos concluye que la mayoría de ellos están plagados de deficiencias en la definición de los constructos implicados (empezando por el término ciencia y el de actitudes) y en la calidad psicométrica de los instrumentos empleados.

Posteriormente, Haladyna y Shaughnessy (1982) resumieron las dificultades que han asolado la investigación sobre actitudes en ciencias: la falta de un marco teórico que fundamente la aplicación adecuadamente contextualizada parece Ia más importante y difícil de solucionar $\mathrm{y}$, hasta cierto punto, la causa de todas las demás dificultades, cuya más visible y notoria consecuencia es la gran cantidad de definiciones de actitudes relacionadas con la ciencia existentes en la bibliografía, pero también la falta de integración que resuma y valore adecuadamente los resultados de tantas investigaciones deslabazadas, la selección de variables fortuitas y los defectos de diseño y análisis en los experimentos realizados, así como los problemas de validez de los instrumentos aplicados para la medida de las actitudes en ciencias.

Una de las revisiones más citadas, que recoge lo más importante deI área, es Ia realizada por Schibeci (1984), aunque restringida a las actitudes relacionadas con la ciencia aparecidas en sólo tres países anglosajones. Desde 1975 el número de publicaciones relacionadas con las actitudes en ciencias alcanza un promedio de unas 30 anuales, observándose un suave crecimiento en los últimos años. Sin embargo, aunque son muy positivos todos los índices cuantitativos que revelan la magnitud del interés por las actitudes entre los investigadores, cuando se analizan las relaciones encontradas entre actitudes y otras variables, los resultados son a veces contradictorios. Es difícil extraer una sintesis clara de la diversidad de resultados, apúntandose como causa fundamental la falta de una base teórica clara que guíe las investigaciones tanto descriptiva como metodológicamente. A ello se añaden los problemas de validez de los instrumentos aplicados y la multiplicidad de los presupuestos teóricos empleados como guía de las investigaciones. Entre los resultados que concitan más coincidencia está la cuestión de género: las mujeres rechazan más las ciencias que los hombres (especialmente las ciencias físicas y, en menor cuantía, la biología). Asimisno, se informa insistentemente de un decrecimiento del interés hacia las ciencias a medida que el estudiante avanza por el sistema educativo; el cual se manifiesta en una escasa elección de las materias y especialidades científicas.

Recientemente, Shrigley y Koballa (1992) revisan la última década de investigación sobre actítudes en ciencias $\mathrm{y}$, a pesar del gran volumen de investigación desarrollado, juzgan que sólo unas pocas conclusiones provisionales pueden considerarse productivas, debido a la falta de un marco teórico que guíe la investigación. Consideran que la psicologia social suministra las teorías básicas para un cambio revolucionario en el enfoque de la investigación. El modelo de Hovland (Hovland, Lumsdaine y Sheffield, 1949), la teoría de la acción razonada de Fishbein y Ajzen (1981) y el modelo de elaboración de Petty y Cacioppo (1986) serían los gigantes, desde cuyos hombros se podría ampliar la visión del horizonte de las actitudes, parafraseando Ia conocida reflexión de Sir Isaac Newton. La mayoría de Ia investigación sobre la modificación de actitudes en ciencia ha estado basada, directa o indirectamente, en el modelo de Hovland; sin embargo, la teoría de la acción razonada (Fishbein y Ajzen, 1975) ha gozado de gran aceptación y ha comenzado a aplicarse en investigaciones recientes referidas a las actitudes en ciencia (Crawley y Coe, 1990; Krynowsky, 1988 y Wareing, 1990).

En resumen, a luz de estas revisiones parecen claras dos recomendaciones fundamentales: en primer lugar, desde el punto de vista teórico se reconoce el papel director que debe jugar la psicología social como marco de referencia en la investigación sobre actitudes; en segundo lugar, desde el punto de vista metodológico debe ponerse 
especial cuidado en la definición de los constructos actitudinales utilizados y su correspondencia precisa con Ios instrumentos empíricos diseñados para la medida, así como en el control de variables empíricas en los diseños experimentales.

\section{FACTORES DE LAS ACTITUDES}

Desde la perspectiva de la psicología social, como marco más general de las actitudes, se puede decir que los temas de interés relacionados con la investigación empírica de las actitudes se pueden resumir en dos grandes apartados: la génesis y cambio de actitudes (métodos más efectivos y variables moduladoras) y las relaciones entre actitudes y conducta (condiciones bajo las que una actitud es una guía efectiva de la conducta); comunes a ambos es el diseño de instrumentos válidos y fiables para la medida de actitudes. Por el contrario, el estudio de las actitudes relacionadas con la ciencia ha sido más descriptiva y centrada en visiones limitadas, y con frecuencia no bjen definidas, del objeto de actitud, debido a la falta de un marco teórico; se revisarán a continuación algunas de las investigaciones realizadas en el área, con especial atención a las variables determinantes de las actitudes consideradas en ellas.

La preocupación por realizar mediciones de las actitudes en ciencia, que sean válidas, ha sido recogida en numerosos trabajos, como muestra de los cuales pueden citarse Ios estudios básicos de Shrigley y Koballa (1984), Bratt (1984), Zeidler (1984) y, en nuestro país, los de Acevedo (1993b), Moreno y Gil (1987) y Ortega y otros (1992), entre diversos autores.

El sexo del alumnado es una de las principales variables estudiadas (Cannon y Simpson, 1985; Erickson y ErickSon, 1984; Handley y Morse, 1984; Hartey y Beall, 1984; Levin y Fowler, 1984; Reid y Tracey, 1985 y Smail y Kelly, 1984) obteniéndose, en general, que los chicos están más interesados en las ciencias que las mujeres, aunque con matices, ya que mientras las preferencias de los hombres se dirigen hacia las ciencias físicas, las mujeres hacen lo propio con los temas biológicos. Kelly (1986) realiza un estudio longitudinal ( 11 y 14 años) con 1.300 alumnos británicos de 10 escuelas, mostrando que las actitudes en ciencias decrecen al cabo del período citado (excepto en biología que crecen), y el programa de intervención para mejorar las actitudes de las chicas produjo resultados positivos, pero pequeños.

La constatación de una progresiva dicotomía social entre dos culturas, de los citsdadanos científicos y los no científicos, y la creciente disminución de la cultura científica y eI progresivo analfabetismo científico en la sociedad, porque los estudiantes eligen cada vez menos asignaturas de ciencias en sus estudios, ha sido otra constante preocupación. Así, Gogolin y Swartz (1992) comparan las actitudes en ciencias entre universitarios científicos (102) y no cientúficos (81) matriculados en un curso de anatomía y fisiología. Los estudiantes de ciencias mostraron actitudes más positivas hacia la ciencia y más bajos niveles de ansiedad que los no científicos; después del curso, ambos grupos mejoraron significativamente sus actitudes. De las entrevistas se dedujo que el ambiente familiar tiene una relación remota con las actitudes en ciencias (tal vez sólo actúa limitando las oportunidades de desarrollo de los aspectos científicos), pero el grupo de iguales tiene una influencia decisiva (de acuerdo con la teoria de Ajzen y Fishbein que postula la influencia social como uno de los determinantes de la conducta). Sin embargo, aunque los estudiantes mejoraban sus puntuaciones de actitudes, los profesores no notaban un aumento real del interés de los estudiantes en la clase, de acuerdo con algunos estudios que preconizan un descenso de las actitudes hacia el aprendizaje de las ciencias a Io largo de la escolarización (Kelly, 1986 y Yager y Pennick, 1986). No obstante, se considera que algunos de estos resultados (p.e. Ia mejora de actitudes después del curso) tiene una validez limitada al tipo de curso realizado (de anatomía y fisiología), ya que otros estudios sugieren que los temas de biología provocan menos rechazo que, por ejemplo, los más difíciles de física.

Shrigley (1990) encuentra que la actitud positiva hacia las ciencias decrece a medida que el estudiante progresa en sus estudios hacia niveles superiores, coincidiendo con otros estudios, incluso entre estudiantes especializados en ciencias (James y Sinith, 1985 y Simpson y Oliver, 1985); sin embargo, Espinosa y Román (1993) encuentran para alumnos universitarios una evolución de Ias actitudes en dientes de sierra.

Debido a la gran disparidad metodológica, con frecuencia, los resultados son conflictivos o divergentes, especialmente en estudios que analizan la influencia de determinadas variables sobre Ias actitudes. Algunas de las variables más estudiadas son las escolares, tales como el rendimiento escolar (Cannon y Simpson, 1985; Friend, 1985; Handley y Morse, 1984; Hartey, Beall y Scharmann, 1985; Okebukola, 1985 y Trout y Crawley, 1985), el trabajo práctico de laboratorio (Ashamn, 1985), el entorno escolar (Hassan, 1985), la influencia de las actitudes de los profesores (Lawrenz, 1985 y Lawrenz y Cohen, 1985) y Ios métodos de enseñanza/aprendizaje de las ciencias (Johnson y Brooker, 1985), pero tambiên han recibido atencion otras variables como los rasgos de personalidad (Hartey, Anderson y Enoch, 1984).

El efecto de programas particulares de ciencias sobre las actitudes varía considerablemente y los resultados no son consistentes: Hufford (1991) describe mejoras en rendimiento y actitudes con un método de aprendizaje cooperativo en un curso introductorio de biología. Hofstein y otros (1990) encuentran que actividades extraescolares de ciencias crean actitudes más positivas hacia la ciencia en la escuela, y Mason y Kahle (1989) con un programa de intervención, que modifica las técnicas y el ambiente de clase para fomentar la participación de las alumnas de ciencias de postsecundaria, mejora las actitudes, la percepción de la ciencia y la experiencia científica, pero Scharf y Schibeci (1990) no encuentran diferencias con un nuevo currículo de transición en ciencias. 
La influencia de las actitudes de los compañeros(Talton, 1985) parece que condiciona las actitudes individuales y algunos sugieren que las actitudes en ciencias influyen en la capacidad de comprensión de los procesos cien. tíficos (Koballa y Crawley, 1985 y Lawrenz y Cohen, 1985). Al mismo tiempo existen investigaciones sobre actitudes referidas a temas científicos concretos, como por ejemplo, la energía (Koballa, 1984a, 1984 b y Calhoun, Shrigley y Showers, 1988), línea desarrollada extraordinariamente por el movimiento CTS, como se verá.

El estudio de Cannon y Simpson (1985) resulta paradigmático como ejemplo de una línea comprensiva que estudia las actitudes en relación con varias variables como la motivación y el rendimiento académico, realizando tres medidas al comienzo, en la mitad y al final de curso en que comenzaban a estudiar ciencia. Los resuitados muestran que las actitudes decrecieron significativamente hasta la mitad de curso y no se recuperaron, en tanto que el rendimiento creció significativamente entre el comienzo y el medio curso y ya no bajó; la motivación en cambio, disminuyó regularmente a lo largo del curso. Los chicos tienen actitudes más positivas hacia la ciencia y alcanzan mejores rendimientos que las chicas, las cuales, sin embargo, están más motivadas para rendir en ciencias. Por grupos, los alumnos de capacidad general fueron los que mostraron los mayores descensos en actituđes hacia las ciencias y motivación de logro.

Las relaciones de las actitudes en ciencias con variables personales (motivación de logro, ansiedad, autoconcep. to en ciencias y autoconcepto general), la familia (cien. cia y general) y el ambiente escolar (clima de clase, currículo, ambiente físico, profesor, compañeros de clase, amigos y la escuela) es estudiada por Talton y Simpson (1986) en alumnos de Ios grados 6 a 10 con una metodología de regresión múltiple. El modelo global consigue explicar un 70-80\% de la varianza total de las actitudes, siendo las variables de ambiente de clase los que poseen una capacidad predictiva mayor.

Simpson y Oliver (1990) presentan un ambicioso estu dio longitudinal sobre actitudes, cuyas principales conclusiones son las siguientes: $\mathrm{La}$ actitud en ciencias decrece a lo largo de cada curso, principalmente entre el inicio y la mitad del curso, y también es constante en los sucesivos grados, con una actitud casi neutra en el último curso. La actitud en ciencias es más consistente y mejor en los hombres que en las mujeres. La evolución de la motivación de logro en el tiempo es marcadamente similar a la de las actitudes; pero la motivación de logro en ciencias fue consistentemente mejor entre las mujeres. Las actitudes de los adolescentes tienen correlaciones altamente positivas con las actitudes de sus amigos. El descenso de las actitudes fue mayor en el grupo de alumnos medios, interpretando esto como una consecuencia de la mayor atención del profesor a los grupos de alumnos más avanzados y más retrasados. Entre las variables personales, escolares y familiares, las varia. bles de la clase de ciencias son las que tienen la mayor influencia sobre las actitudes en ciencias. Las variables individuales son los mejores predictores del rendimiento en ciencias y se obtiene una relación más intensa actitudes/rendimiento que en otros estudios anteriores. Las actitudes en ciencias tienen un papel clave en la determinación de la cantidad de exposición a cursos de ciencia que selecciona un estudiante.

La investigación sobre las actitudes en ciencia en nuestro país es menor y relativamente reciente, pero en moderado crecimiento (Vázquez y Manassero, 1995). La mayoría de trabajos se centran en la actitud hacia el aprendizaje de la ciencia (Acevedo, 1993a; Arana, Escudero, Barnett, 1993; Espinosa y Román, 1991, 1993; Garcés y Palacios, 1985; Gómez y Cervera, 1989; Moreno y Gil, 1987; Ortega et al. 1992; Serrano, 1988 y Soneira, Lires y Pizarro, 1993), y han prestado menos atención al análisis de las actitudes propiamente científicas (Acevedo, 1993b y Serrano, 1986) y a los temas ciencia-técnicasociedad (Álvarez, Soneira y Pizarro, 1993; Guasch, de Manuel y Grau, 1993 y Solbes y Vilches, 1989, 1992), mientras otros estudian las actitudes de los profesores (Escudero y Lacasta, 1984 y Gil, Martínez y Senent, 1987) o realizan una revisión del complejo panorama del campo de las actitudes (Escudero, 1985), sin olvidar la pionera revisión/recopilación bibliográfica realizada en su día por Soler (1986).

En suma, la muestra ofrecida revela suficientemente la complejidad de los estudios y los resultados, muy dependientes de variables situacionales específicas de cada estudio, aunque se puede apreciar un gran esfuerzo para superar las dificuitades teóricas, metodológicas y de instrumentación.

\section{EL CONCEPTO DE ACTITUDES RELA- CIONADAS CON LA CIENCIA}

Uno de los problemas específicos de las actitudes en la educación en ciencias es la confusión conceptual, es decir, la falta de claridad en la definición del objeto de la actitud. Así, se ha criticado muchas veces (Rennie y Parker, 1984) que las actitudes a medir no se definían con suficiente precisión, de modo que el significado de los constructos que se proponen teóricamente y los constructos que miden los cuestionarios no resultan coincidentes (falta de validez).

Una primera precisión a la definición de la actitud se encuentra en la revisión de Aiken y Aiken (1969), quienes señalan tres significados principales asignados al constructo actitud en ciencias: actitudes hacia la ciencia, actitudes hacia los científicos y actitudes hacia el método científico. Posteriormente, Garđner (1975) sugirió dos categorías principales para las actitudes relacionadas con la ciencia, distinguiendo entre actitudes hacia la ciencia y actitudes científicas, que desde entonces se hizo clásica, siendo admitida por muchos (Laforgia, 1988 y Schibeci, 1983 y Wareing, 1990), para abordar la naturaleza claramente multidimensional de las actitudes relacionadas con la ciencia (Jones y Butts, 1983). 
Las actitudes hacia la ciencia serían las disposiciones, tendencias o inclinaciones a responder hacia todos los elementos (acciones, personas, situaciones o ideas) implicados en el aprendizaje de la ciencia (Gardner, 1975a). Se reconocen tres componentes principales: el interés por los contenidos de la ciencia (sosos/aburridoso interesantes/atractivos); y las actitudes hacia los científicos (personas) y su trabajo; y las actitudes hacia los logros de la ciencia desde su ambivalencia en la responsabilidad social (energía y armasnucleares, fertilización in vitro, contaminación industrial, etc.). Las actitudes hacia la ciencia subrayan, sobre todo, el aspecto afectivo de la actitud frente al carácter más cognitivo de las denominadas actitudes científicas.

Las actitudes científicas serían el conjunto de rasgos emanados de las características que el método científico impone a las actividades de investigación científica realizadas por los científicos, como por ejemplo, racionalidad, curiosidad, disposición a cambiar el juicio, imparcialidad, pensamiento crítico, honradez y objetividad, humildad, respeto por la naturaleza y la vida, escepticismo, creatividad... Aunque algunos autores han intentado defínir el número de componentes independientes de las actitudes científicas, el grado de solapamiento entre ellas hace difícil su diferenciación ciara. Gauld y Hukins (1980) propusieron tres grupos para clasificar Ias actitudes cientifficas: actitud general hacia las ideas y la información, actitudes relacionadas con la evaluación de las ideas y la información, y compromiso con creencias científicas específicas. Sin embargo, puesto que la filosofía y epistemología, que contribuyen a clarificar la naturaleza del método científico, han sido dialécticamente variables a lo largo de la historia, esta problemática se trasiada a la definición de las actitudes científicas, Io que las convierte en un área cambiante y con un grado mayor de provisionalidad.

Otro problema añadido en la evaluación de las actitudes científicas es que debe tenerse en cuenta el riesgo -siempre presente en la medición de actitudes-de medir el mero conocimiento de las mismas (p.e., reconocer la honradez como rasgo de los científicos) en lugar de medir la adhesión personal a estos valores (p.e., aceptación personal de la honradez), puesto que las actitudes científicas poseen un carácter más cognitivo que las actitudes hacia la ciencia, de perfil más afectivo y sentimiental.

Hodson (1985) sostiene que la escuela debería ser el elemento fundamental en crear una actitud equilibrada en los ciudadanos y distingue los siguientes tipos de actitudes en ciencias: actitud sobre la ciencia y su imagen pública, actitud sobre los métodos de la ciencia, actitud sobre las actitudes científicas, actitud sobre las implicaciones sociales y ambientales de la ciencia y actitud sobre la enseñanza de las ciencias.

En resumen, todos estos estudios revelan un esfuerzo por alcanzar análisis bien fundados de las actitudes y son coincidentes en reconocer la multidimensionalidad del constructo. Por tanto, existen múltiples objetos de actitud relacionados con la ciencia, que van más allá del limitado concepto de actitud hacia el aprendizaje de la ciencia, y que, para no confundirlo con las etiquetas sugeridas en estudios anteriores, se denominará actitudes (en plural, para subrayar su multipticidad) relacionadas con la ciencia.

\section{LA PERSPECTIVACIENCIA-TECNOLOGÍA- SOCIEDAD: APORTACION A LAS ACTI. TUDES}

En los úttimos años las interrelaciones entre la ciencia, la tecnología y la sociedad emergen como un área de interés para la investigación en didáctica de las ciencias, conformando un movimiento denominado Ciencia-Tecnología-Sociedad (CTS), y que además enfatizan la educación de las actitudes relacionadas con la ciencia como una parte importante de la alfabetización científica. Ciencia y técnica comparten métodos y temas de investigación comunes y, de hecho, a lo largo de la historia, ha existido una fecundación mutua y productiva entre ambas. Ahora bien, en los currículos escolares de ciencias ha sido habitual despreciar la dimensión técrica en favor de unos conocimientos científicos idealizados, desconectados de lo que los alumnos viven cada día y que, por tanto, no resultan funcionales y útiles para muchas personas. La recuperación de los aspectos tecnológicos para el currículo que propone el enfoque CTS supone hacer una ciencia de la vida diaria 0 , por lo menos, muy relacionada con el entorno habitual de los alumnos. Otro aspecto central es la consideración de los aspectos sociales y su interacción con la ciencia y la tecnología. Se subraya que el desarrollo científico y tecnológico ha tenido y tiene un importante papel en muchas transformaciones sociales, pero también que las circunstancias sociales (cultura, pensamiento, recursos económicos ...) son las que hacen posible y condicionan la evolución y el avance de la ciencia y la tecnología. El análisis de los currículos ejemplares CTS desarrollados en EE UU en los últimos años evidencia la existencia de dos enfoques básicos para la educación CTS (Rosenthal, 1989): un enfoque sobre cuestiones sociales, es decir, sobre temas con relevancia social que caen dentro de la órbita de la ciencia y la tecnología, como la gestión de recursos, la conservación del medio ambiente, las fuentes de energía, el crecimiento demográfico, la energía nuclear, la ingeniería genética, etc; y un enfoque sobre los aspectos sociales de la ciencia, es decir, el análisis de la interacción entre C y T y otras disciplinas sociales (y viceversa), tales como la filosofía (epistemología y ética), sociología (posibilidades y limitaciones de la C y T para resolver los problemas de la sociedad), historia (relaciones mutuas historia con Cy T), política (políticas globales, políticas científicas, decisiones políticas, defensa nacional), economía (industria, consumo, empleo).

Desarrollar una adecuada comprensión de la naturaleza de la ciencia, o sea, de los valores y suposiciones propias del desarrollo del conocimiento científico, es un objetivo de la educación en ciencias que concita un gran acuerdo universal y es enfatizado desde la perspectiva 
CTS (Aikenhead, 1979) por la naturaleza dialéctica de la interacción entre $\mathrm{C}$ y $\mathrm{T}$ y las disciplinas sociales. El falsacionismo de Popper (1977), el evolucionismo revolucionario de Kuhn (1962), el progreso de los programas científicos de Lakatos (1983), Ia posición ácrata de Feyerabend (1982), el evolucionismo racional de Toulmin (1972) y el racionalismo de Bunge (1976, 1980) representan distintas posiciones de análisis y crítica que han contribuido a acotar y precisar los aspectos esenciales de la ciencia y la metodología científica. En síntesis, las aportaciones anteriores subrayan la perspectiva evoIucionista de la ciencia, como un conocimiento siempre crítico, inacabado, dinámico y revisable $\mathrm{y}$, por tanto, siempre provisional e incierto. Además, como toda actividad humana y social, la ciencia es una empresa colectiva, con crisis y errores, sometida a los mismos condicionamientos sociales que otras actividades humanas, por la política de desarrollo científico, las relaciones de poder de los grupos sociales y de la comunidad científica e influida por las ideologías dominantes y las creencias de las personas y de las sociedades, de las cuales es una muestra evidente la división de los roles sociales entre hombre y mujer, que origina una menor participación de las mujeres en la investigación científica, respecto a los hombres (infrarepresentación femenina en la ciencia). La educación en ciencias debe producir sobre todos estos aspectos actitudes coherentes y concordantes con la naturaleza de la ciencia, las relaciones $\mathrm{C}$ y $\mathrm{T}$ y sociedad y las diversas cuestiones sociales.

La imagen devaluada y negativa de la ciencia y los científicos, a través del estereotipo del científico loco y chiflado (Schibeci, 1986), tiene como primera consecuencia una actitud de desapego o rechazo hacia la ciencia y los científicos, que tiene varios efectos macroscópicos indeseables para un país: el primero sería la poca implicación de alumnos en estudios científicos, lo cual afectaría a la situación general de un país, que puede llegar a ser perjudicado o puesto en riesgo (National Commission, 1983) al no disponer de científicos y técnicos en número suficiente -o bien preparados- para satisfacer las necesidades del sistema económico-productivo general; el segundo efecto sería la menor representación de las mujeres en los ámbitos científicos y técricos, tanto escolares (donde tienen un rendimiento diferenciado respecto a los hombres y eligen menos materias o itinerarios de ciencias) como profesionales; el tercer efecto negativo seria la formación de ciudadanos con una alfabetización científica deficiente y sesgada, hecho de enorme trascendencia a la hora de ejercer derechos y asumir responsabilidades en una sociedad democrática, especialmente en los asuntos de trascendencia social relacionados con la ciencia y la tecnología.

En resumen, la perspectiva CTS aporta y resalta aspectos claramente actitudinales e impulsa la necesidad de promover actitudes adecuadas, pero no sesgadas, en relación con los aspectos sociales, Ia imagen y naturaleza de la ciencia y los científicos para la educación en ciencias. Desde sus postulados dialécticos, interdisciplinares e interactivos socialmente, el enfoque CTS resulta decisivo, pues aporta significativos componentes a las actitudes relacionadas con la ciencia (Acevedo, 1993a; Solbes y Vilches, 1989), tanto desde la perspectiva de las cuestiones con relevancia social, hacia las cuales debe conseguirse una actitud decididamente crítica y positi$\mathrm{va}$, como desde la perspectiva de los aspectos sociales de la ciencia. El planteamiento CTS es una fuente inestimabie y actualizada de nuevas actitudes relacionadas con la ciencia que resalta y refuerza una perspectiva humanista y social de la educación en ciencias.

\section{UNA TAXONOMÍA PARA LAS ACTITUDES RELACIONADAS CON LA CIENCIA}

A la vista de la revisión realizada ha quedado patente la naturaleza multidimensional del constructo actitudes relacionadas con la ciencia, de modo que no cabe hablar de una actitud en ciencia o hacia la ciencia, en singular, sino de múltiples y diferenciados constructos de actitudes relacionadas con Ia ciencia, en plural. Como consecuencia, Ia existencia de esta pluralidad de actitudes potenciales hace absolutamente necesario especificar y definir el tipo de actitud relacionada con la ciencia, especialmente en los estudios de investigación sobre estos temas, no sólo para cumplir con un requerimiento metodológico imprescindible, sino por un elemental sentido de la clarificación y precisión en la comunicación científica. Calhoun, Shrigley y Showers (1988) ofrecen un ejemplo para definir correctamente el constructo que miden (actitudes hacia el uso de la energía nuclear para producir electricidad) diferenciándolo de otros próximos como las actitudes hacia la proliferación nuclear, el empleo de isótopos radiactivos en medicina o similares.

Sin embargo, la complejidad requiere esfuerzos de sintesis que ayuden en la comprensión, sin pérdida de precisión. Por ello, como colofón de este artículo, y a modo de propuesta, teniendo en cuenta las distintas aportaciones analizadas, se ofrece una taxonomía de las actitudes relacionadas con la ciencia, con tres grandes grupos, dentro de los cuales se consideran algunas subdivisiones. Como una aportación general del movimiento CTS consideraremos que la referencia a la ciencia, debe contemplarse como una referencia a la ciencia y la tecnología(C y T); por ello, en los párrafos siguientes las referencias a la ciencia y lo científico se entienden extendidas a la tecnología y lo técnico, aunque no se explicitarán siempre para no complicar la redacción.

\section{Actitudes relacionadas con la enseñanza/aprendizaje de la Ciencia y Tecnología}

1. Elementos escolares de la $C$ y $T$. El objeto de estas actitudes son los aspectos de la $\mathrm{C}$ y $\mathrm{T}$ tal como se perciben por los alumnos en el contexto escolar, a través de los elementos curriculares (objetivos, contenidos, evaluación, asignaturas, itinerarios...), los procesoseducativos, las personas intervinientes (profesores, compañeros), etc. Aunque para un profesor de $\mathrm{C}$ y $\mathrm{T}$ estas actitudes tienen un interés obvio, la $\mathrm{C}$ y $\mathrm{T}$ como objeto directo de la actitud se encuentran muy mediatizadas por 
las variables situacionales de la escuela, de modo que el objeto de actitud no es la C y T misma, sino el tipo de C $\mathrm{y} T$ ofrecido por una escuela o un profesor específico; su trascendencia, sin embargo, es muy grande porque para muchos ciudadanos es su único contacto con la $\mathrm{C}$ y $\mathrm{T}$.

2. Los productos del aprendizaje de la $C$ y $T$. El objeto de estas actitudes serían los logros conseguidos como consecuencia de la intervención escolar, tales como la alfabetización científica de los ciudadanos, la opción por estudios y materias científicos y técnicos, las interacciones entre las dos culturas (científicos y no científicos), la utilidad y funcionalidad de la $\mathrm{C}$ y $\mathrm{T}$ aprendida para la vida diaria (educación para el consumo, la salud, medio ambiente, etc.).

\section{Actitudes relacionadas con fas interacciones entre Sociedad y Ciencia y Tecnología}

3. La imagen social de la $C$ y $T$. El objeto de estas actitudes son los aspectos sociológicos de la $\mathrm{C}$ y $\Upsilon$, como sistema, en interacción con la sociedad y viceversa; por tanto, aquí se podrían encuadrar los temas de control de la $\mathrm{C}$ y $\mathrm{T}$ por la sociedad, las relaciones de la $\mathrm{C}$ y $\mathrm{T}$ con la industria civil y militar, la responsabilidad social de la $\mathrm{C}$ y $T$, la toma de decisiones en asuntos científicos que afectan a la sociedad, las consecuencias positivas y negativas de las aplicaciones científicas, contribuciones de la $\mathrm{C}$ y $\mathrm{T}$ al bienestar social, al pensamiento, a la cultura, etc.

4. Temas específicos de $C$ y $T$ con incidencia social. EI objeto de actitud serían temas concretos y específicos de C y $\tau$ cuya importancia radica en su trascendencia social: preservación del medio ambiente (lluvia ácida, vertidos industriales ...), proliferación nuclear, atmósfera y polución (decrecimiento de la capa de ozono, efecto invernadero...), crecimiento demográfico, recursos alimenticios y hambre, tecnología de guerra, agua, escasez energética, sustancias peligrosas, salud y enfermedades, uso de la tierra, reactores nucleares, extinción de animales y plantas y recursos minerales, fuentes y recursos energéticos, ingeniería genética, etc.

\section{Actitudes relacionadas con el conocimiento cien- tífico y técnico}

5. Las características de los científicos. El objeto de este grupo de actitudes son los aspectos relacionados personalmente con los científicos, como la motivación en su trabajo, los valores del trabajo científico (honradez, objetividad, escepticismo, apertura...), la ideología de los científicos, las cualidades personales, los efectos de género en la $\mathrm{C}$ y $\mathrm{T}$, etc.

6. La construcción colectiva del conocimiento científi-
co. El objeto de estas actitudes serían las relacionadas
con la toma de decisiones en la construcción del conoci-
miento científico: la competencia entre científicos, la
comunicación profesional, el consenso y el desacuerdo
entre los científicos, la lealtad al grupo de investigación, la C y T pública y privada, la influencia de los individuos en el conocimiento, la influencia de la sociedad sobre los científicos, etc.

\section{Actitudes relacionadas con la naturaleza del conoci-} miento científico. Los objetos de actitud sería los procesos y productos propios del conocimiento científico, tales como la naturaleza de las observaciones, los esquemas de clasificación, las hipótesis, las teorías, las leyes, los modelos, la precisión y la incertidumbre, la naturaleza tentativa y provisional, el razonamiento lógico, el estatus epistemológico, etc.

No se pretende ofrecer con esta taxonomía una visión acabada y simplista de las actitudes relacionadas con la C y T; sino al contrario, la primera cuestión que salta a la vista es la relación y el solapamiento entre las diferen. tes categorias, que revelan toda la complejidad y multidimensionalidad del tema de las actitudes relacionadas con la C y T, a pesar de lo cual o gracias a ello, según se mire, creemos que es un área llena de desafíos y de atractivo en la enseñanza/aprendizaje de las ciencias. Desde una perspectiva puramente conceptual, esta taxonomía supone una superación del concepto reducido de actitud hacia la ciencia como actitud hacia el aprendizaje de la ciencia, poniendo de manifiesto, por el contrario, su mayor riqueza y variedad, en correspondencia con la riqueza y variedad de valores que subsumen las actividades científicas.

Por otro lado, es bien conocida la existencia de prejuicios y reticencias respecto a la educación de las actitudes relacionadas con la $\mathrm{C}$ y $\mathrm{T}$ en la comunidad docente y científica. Esta postura es tanto más contradictoria y relevante si se considera que continúa subsistiendo, incluso entre personas bien informadas en didáctica de la ciencias, y además en un momento en que se está implantando en nuestro país una reforma educativa, una de cuyas innovaciones es introducir en el currículo escolar las actitudes y los valores como contenidos independientes y autónomos, no subordinados a otros contenidos, aunque lógicamente interrelacionados con los contenidos conceptuales y procedimentales. En esta línea no deja de ser sorprendente ver desarrollos y secuenciaciones curriculares de ciencias, incluso promovidos por el propio Ministerio de Educación, que relegan la educación de las actitudes a una parte insignificante del discurso diđáctico y, en algún caso, hasta se eliminan sistemáticamente de las propuestas de secuenciación. Los profesores de C y $T$ deberían asumir decididamente la educación integrada de las actitudes como un objetivo importante de la enseñanza de la C y $\mathrm{T}$ para formar ciudadanos (especialmente como usuarios y consumidores), que adquieran una opinión y alfabetización científica y tecnológica más acorde con las exigencias de la sociedad actual y con las pautas críticas y humanizadoras que caracterizan un enfoque de la educación en ciencias enmarcado en el paradigma CTS; pero también para formar científicos más autocríticos y conscientes del papel social y humano que juega la ciencia en nuestra sociedad, en línea con las sugerencias de $\mathrm{G}$. Moliner y $\mathrm{F}$. Rañada (1994). En esta línea, la educación de las actitudes relacionadas con la ciencia se convertiría en elemen- 
to esencial de una orientación humanista y humanizada de la ciencia para todos los alumnos, sin distinción de su especialización científica o no científica. Esperamos que estas reflexiones ayuden a promover una educación de las actitudes más efectiva y a conseguir una educación en ciencia que, por esta vía de la educación de las actitudes relacionadas con la ciencia, logre una verdadera alfabetización cientifica de base crítica y humanista para todos los ciudadanos.

\section{NOTA}

- Estudio financiado por el Centro de Investigación Documentación y Evaluación (CIDE) del MEC a través de las Ayudas a la Investigación Educativa de 1992.

\section{REFERENCIAS BIBLIOGRÁFICAS}

ACEVEDO, J.A. (1993a). ¿Qué piensan los estudiantes sobre la ciencia? Un enfoque CTS. Enseñanza de las Ciencias, Vol. exłra, IV Congreso Internacional sobre investigación en la didáctica de las ciencias y las matemáticas, pp. 11-12. Barcelona 13-16 septiembre.

ACEVEDO, J.A. (1993b). Actitudes hacia el aprendizaje de las ciencias físicas, naturales y matemáticas en el BUP y COU. Un estudio sobre tres dimensiones. Enseñanza de las Ciencias, Vol. extra, IV Congreso Internacional sobre investigación en la didáctica de las ciencias y las matemáticas, pp. 13-14. Barcelona, 13-16 septiembre.

AIKEN, R.L. y AIKEN, D.R. (1969). Recent research on attitudes concerning science. Science Education, 53, pp. 295-305.

AIKENHEAD, G. (1979). Science: A way of knowing. The Science Teacher, 46, pp. 23-25.

AJZEN, 1. y FISHBEIN, M. (1977). Attitude-behavior relations: a theoretical analysis and review of empirical research. Psychological Bulletin, 84, pp. 888-918.

ÁlVAREZ, M., SONEIRA, G., y PIZARRO, 1. (1993). Cómo percibe el alumnado algunas interacciones entre cienciatecnología-género-sociedad. Enseñanza de las Ciencias, Vol. extra, IV Congreso Internacional sobre investigación en la didáctica de las ciencias y las matemáticas, $p p$. 19-20. Barcelona 13-16 septiembre.

ARANA, J., ESCUDERO, T., GARCÉS, R. y PALACIOS, C. (1985). Imagen de las asignaturas de ciencias en la transición de la educación básica a la secundaria. Enseñanza de las Ciencias, Vol. extra, III Congreso, p. 20.

ASHMAN, A. (1985). Chemistry in schools-past, present and future. Part 2. School Science Review, 67, pp. 277-284.

BARNETT, D. (1993). Actitud hacia la ciencia y la enseñanza de la ciencia. Enseñanza de las Ciencias, Vol. extra, IV Congreso Internacional sobre investigación en la didáctica de Ias ciencias y las matemáticas, pp. 27-28. Barcelona 13. 16 septiembre.

BRATT, M. (1984). Further Comments on the Validity Studies of Attitude Measures in Science Education. Journal of Research in Science Teaching, 21(9), p. 951.

BUNGE, M. (1976) La investigación científica. Barcelona: Ariel. BUNGE, M. (1980). Epistemología. Barcelona: Ariel.
CALHOUN, L., SHRIGLEY, R.L. y SHOWERS, D.E. (1988). Designing the Nuclear Energy Attitude Scale. Science Education, 72(2), pp. 157-174.

CANNON, R.K., Jr. y SIMPSON, R.D. (1985). Relationships among Attitude, Motivation, and Achievement of Ability Grouped, Seventh-Grade, Life Science Students. Science Education, 69(2), pp. 121-138.

CRAWLEY, F.E y COE, A.E. (1990). Determinants of middle school students' intentions to enroll in a high school science course: An application of the theory of reasoned action. Journal of Research in Science Teaching, 27, pp. 461-476.

ERICKSON, G.L. y ERICKSON, L.J. (1984). Females and science achievement: Evidence, explanations and implications. Science Education, 68(2), pp. 63-89.

ESCUDERO, T. y LACASTA, E. (1984). Las actitudes científtcas de los futuros maestros en relación con sus conocimientos. Enseñanza de las Ciencias, 2(3), pp. 175-180.

ESCUDERO ESCORZA, T. (1985). Las actitudes en la enseñanza de las ciencias: un panorama complejo. Revista de Educación, 278 , pp. 5-25.

ESPINOSA, J, y ROMÁN, T. (1991). Actitudes hacia la ciencia y asignaturas pendientes: dos factores que afectan al rendimiento en ciencias. Enseñanza de las Ciencias, 9(2), pp. 151-154.

FEYERABEND, P.K. (1982). Contra el método. Madrid: Tecnos.

FISHBEIN, M. y AJZEN, I. (1975). Belief, Attitude, Intention, and Behavior. Reading, Mass.: Addison-Wesley.

FISHBEIN, M. y AJZEN, I. (1981). Acceptance, yielding and impact: cognitive processes in persuasion, en Petty, R.E. Ostrom, T.M. y Brock, T.C. (eds.), Cognitive Responses in Persuasion. Hillsdale, N.I.: Erlbaum.

FRIEND, H. (1985). The effect of Science and Mathematics Integration On Selected Seventh Grade Students' Attitudes. School Science and Mathematics, 85(6), pp. 453-461.

GARCÍA MOLINER, F. y F. RAÑADA, A. (1994). Invitación a la autocrítica. Revista Española de Física, 8(3), pp. 2-4.

GARDNER, P.L. (1975). Attitudes to science: A review. Studies in Science Education, 2, pp. 1-41.

GAULD, C.F y HUKINS, A.A. (1980). Scientific attitudes: A review. Studies in Science Education, 7, pp. 129-161. 
GIL, D., MARTÍNEZ, J. y SENENT, F. (1987). Actitudes y expectativas deI profesorado ante la resolucion de problemas de Física. Enseñanza de las Ciencias, Vol. extra, II Congreso, pp. 203-205.

GOGOLIN, L. y SWARTZ, F. (1992). A quantitative and qualitative inquiry into the attitudes toward science of nonscience college students. Journal of Research in Science Teaching, 29, pp. 487-504.

GÓMEZ, C. y CERVERA, S. (1989). Actitudes y cambio conceptual en la educación ambiental. Enseñanza de las Ciencias, Vol. extra, III Congreso, pp. 209-211.

GUASCH, E., de MANUEL, J., y GRAU, R. (1993). La imagen de la ciencia en alumnos y profesores. La influencia de los medios de comunicación. Enseñanza de las Ciencias, Vol. extra, IV Congreso Internacional sobre investigación en la didáctica de las ciencias y las matemáticas, pp. 77-78. Barcelona 13-16 septiembre.

HALADYNA, T. y SHAUGHNESSY, J. (1982). Attitudes towards science: A quantitative synthesis. Science Education, 66, pp. 547-563.

HANDLEY, H.M. y MORSE, L.W. (1984). Two-year study relating adolescents' self-concept and gender role perceptions to achievement and attitudes toward science. Journal of Research in Science Teaching, 21(6), pp. 559-607.

HARTY, H., ANDERSON, H.O. y ENOCHS, L.G. (1984). Exploring Relationship among Elementary School Students'Interest in Science, Attitudes toward Science and Reactive Curiosity. School Science and Mathematics, pp. 84(4), 308-315.

HARTY, H. y BEALL, D. (1984). Attitudes toward Science of Gifted and Nongifted fifth graders. Journal of Research in Science Teaching, 21(5), pp. 483-488.

HARTY, H., BEALL, D., y SCHARMANN, E. (1985). Relationship between Elementary School Students'Science Achievement and Their Attitudes Toward Science. School Science and Mathematics, 85(6), pp. 472-479.

HASSAN, O.E. (1985). An Investigation into Factors Affecting Attitudes toward Science of Secondary School Students in Jordan. Science Education, 69(1), pp. 3-18.

HODSON, D. (1988). Toward a philosophically more valid science curriculum. Science Education, 72, pp. 19-40.

HOFSTEIN, A. et al. (1990). Attitudes towards School Science: A Comparison of Participants and Nonparticipants in Extracurricular Science Activities. School Science and Mathematics, 90, pp. 13-22.

HOVLAND, C.I. LUMSDAINE, A.A. y SHEFFIELD, F.D. (1949). Communtcation and persuasion. New Haven: Yale University Press.

HUFFORD, T.L. (1991). Increasing Academic Performance in an Introductory Biology Course. BioScience, 41, pp. $107-108$

JAMES, R.K. y SMITH, S. (1985). Alienation of Students from Science in Grades 4-12. Science Education, 69, pp. 39-45.

JOHNSON, R. y BROOKER, C. (1985). The effects of controversy, concurrence seeking, and individualistic learning on achievement and attitude change. Journal of Research in Science Teaching, 22(3), pp. 197-205.

IONES, B. y BUTTS, B. (1983). Development of a set of scales to meastre selected scientific attitudes. Research in Science Education, 13, pp. 133-140.
KELLY, A. (1986). The development of girls' and boys' attitudes to science: A longitudinal study. European Journal of Science Education, 8(4), pp. 399-412.

KELLY, A. (1988). Sex Stereotypes and School Science: A three year follow up. Educational Studies, 14(2), pp. 151163.

KOBALLA, T.R. (1984a). Designing a Likert-Type scale to assess attitudes toward energy conservation: a nine steep process. Journal of Research in Science Teaching, 21(7), pp. 709-723.

KOBALLA, T.R. (1984b). Changing attitudes toward energy conservation: the effect of development advancement on the salience of persuasive comunications. Journal of Research in Science Teaching, 21(6), pp. 659-668.

KOBALLA, T.R. y CRAWLEY, F.E. (1985). The influence of Attitude on Science Teaching and Learning. School Science and Mathematics, 85(3), pp. 222-231.

KRYNOWSKX, B.A. (1988). Ptoblems in Assessing Student Attitude in Science Education: A Partial Solution. Science Education, $72(4)$, pp. 575-584.

KUHN, T.S. (1962). The Structure of Scientific Revolutions. Chicago: University of Chicago Press. México: Fondo de Cultura Económica, 1978.

LAFORGIA, J. (1988). The affective domain related to science education and its evaluation. Science Education, 72, pp. $407-421$.

LAKATOS, I. (1983). La metodología de los programas de investigación cientifica. Madrid: Alianza Editorial.

LAWRENZ, F. (1985). Impact on a Five Week Energy Educationt Program on Teacher Beliefs and Attitudes. School Science and Mathematics, 85(1), pp. 27-36.

LAWRENZ, F. y COHEN, H. (1985). The effects of Methods Classes and Practise Teaching on Student Attitudes toward Science and Knowledge of Science Processes. Science Education, 69(1), pp. 105-113.

LEVIN, J. y FOWLER, H.S. (1984). Sex, grade, and course differences in attitudes that are related to cognitive performance in secondary science.Journal of Research in Science Teaching, 21(2), pp. 151-166.

MASON, C.L. y KAHLE, J.B. (1989). Student Attitudes toward Science and Science-Related Careers: A Program Designed to Promote a Stimulating Gender-Free Leaming Environment. Journal of Research in Science Teaching, 26, pp. 25-39.

MORENO, M.D. y GIL, D. (1987). La medida de los estudiantes de BUP hacia la física. Enseñanza de las Ciencias, Vol. extra, II Congreso, pp. 268-270.

NCEE. National Commission for Excellence in Education. (1983). A nation at risk. Washington, D.C.: National Institute of Education.

OKEBUKOLA, P.A. (1985). Science laboratory behavior strategies of students relative to performance in and attitude to laboratory work. Journal of Research in Science Teaching, 22(3), pp. 221-232.

ORMEROD, M.B. y DUCKWORTH, D. (1975). Pupil's attitudes to science: a review of research. Windsor: NFER Publishing Co.

ORTEGA, P., SAURA, J.P., MÍNGUEZ, R., GARCÍA, A. y MARTÍNEZ, D. (1992). Diseño y aplicación de una escala de actitudes hacia el estudio de las ciencias experimentales. Enseñanza de las Ciencias, 10, pp. 295-303. 
PETTY, R.E. y CACIOPPO, J.T. (1986). Communication and persuasion: Central and peripheral routes to attitude change. Nueva York: Springer-Verlag.

POPPER, K.R. (1977). La lógica de la investigación científica. Madrid: Tecnos.

REID, D.J y TRACEY, D.C. (1985). The evaluation of a school science syllabus through objectives and attitudes. European Journal of Science Education, 7(4), pp. 375-386.

RENNIE, L.J. y PARKER, L.H. (1984). Problems in the interpretation of attitude data, pp. 718-725. Perth: AARE Conference.

ROSENTHAL, D.B. (1989). Two approaches to ScienceTechnology-Society (STS) Education. Science Education, 73(5), pp. 581-589.

SCHARF, P.F. y SCHIBECI, R.A. (1990). The Influence of a «Transition Science» Unit on Student Attitudes. Research in Science and Technological Education, 8, pp. 79-88.

SCHIBECl, R.A. (1983). Selecting approprtate attitudinal objectives for school science. Science Education $67, \mathrm{pp}$. $595-603$.

SCHIBECI, R.A. (1984), Attitudes to science: An update. Studies in Science Education, 11, pp. 26-59.

SCHIBECI, R.A. (1986). Images of science and scientists and science education. Science Education, 70, pp. 139-149.

SERRANO, T. (1986). La imagen de los científicos en Ios alumnos al finalizar el ciclo medio. Documentos IEPS, Monografias, 1. Madrid: IEPS.

SERRANO, T. (1988), Actitudes de los alumnos y aprendizaje de las ciencias. Un estudio longitudinal. Investigación en la Escuela, 5, pp. 29-38.

SHRIGLEY, R.L. (1990). Attitude and Behavior Are Correlates. Journal of Research in Science Teaching, 27, pp. 97-113.

SHRIGLEY, R.L. y KOBALLA, T.R. (1984). Attitude measurement: judging the emotional intensity of Likerttype science attitude statements. Journal of Research in Science Teaching, 21(2), pp. 111-118.

SHRIGLEY, R.L. y KOBALLA JR., T.R. (1992), A decade of attifude research based on Hovland's learning model. Science Education, 76(1), pp. 17-42.

SIMPSON, R.D. y OLIVER, J.E. (1985). Attitude Toward Science and Achievement Motivation Profiles of Male and Female Science Students in Grades Six through Ten. Science Education, 69(4), pp. 511-526.

SIMPSON, R.D. y OLIVER, J.E. (1990). A Summary of Major Influences on Attitude Toward an Achievement in Science Among Adolescent Students. Science Education, 74(1), pp. 1-18.
SOLBES, J. y VILCHES, A. (1989). Interacciones ciencia/ técnica/sociedad: un instrumento de cambio actitudinal. Enseñanza de las Ciencias, 7(1), pp. 14-20.

SOLBES, J. y VILCHES, A. (1992). El modelo constructivista y las relaciones ciencia/técnica/sociedad (CTS). Enseñanza de las Ciencias, 10, pp. 181-186.

SMAIL, B. y KELLY, A. (1984). Sex differences in Science and Technology among eleven year old schoolchildren II: attitudes. Research in Science Technology Education, 2, pp. $87-106$.

SOLER, J.B. (1986). La actitud de los alumnos hacia la ciencia y su enseñanza. Enseñanza de las Ciencias, 4, pp. 174-175.

SONEIRA, G., LIRES, M.A. y PIZARRO, I. (1993). Actitudes del alumnado ante las materias optativas de ciencias y letras. Diferencias de género. Enseñanza de las Ciencias, Vol. extra, IV Congreso Internacional sobre investigación en la didáctica de las ciencias y las matematicas, pp. 137-138, Barcelona 13-16 septiembre.

TALTON, E.L. y SIMPSON, R.D. (1985). Relationships between Pair and Individual Attitudes toward Science among Adolescent Students. Science Education, 69(1), pp. 19-24.

TALTON, E.L. y SIMPSON, R.D. (1986). Relationships of attitudes toward self, family, and school with attitude toward science among adolescents. Science Education, 70 , pp. 365 374.

TOULMIN, S. (1977). La comprensión humana: I. El uso colectivo y la evolución de los conceptos. Madrid: Alianza Universidad.

TROUT, J.S. y CRAWLEY, F.E. (1985). The effects of matching instructional strategy with selected student characteristics on ninth grade physical science. Journal of Research in Science Teaching, 22, pp. 407-419.

VÁZQUEZ,A. y MANASSERO, M.A. (1995).Actitudes hacia la ciencia y sus relaciones con la tecnologia y la sociedad en alumnos de todos los niveles educativos. Memoria final de investigación. Madrid: MEC-CIDE.

WAREING, C. (1990). A Survey of Antecedents of Attitudes Toward Science. Journal of Research in Science Teaching, 27(4), pp. 371-386

YAGER, R.E. y PENICK, E.J. (1986). Perceptions of four age groups towards science classes, teachers and the value of science. Science Education, 70, pp. 355-364.

ZEIDLER, D.L. (1984). Thirty studies involving the "Scientific Attitude Inventory": what confidence can we have in this instrument. Journal of Research in Science Teaching, 21(3), pp. 341-342. 